\title{
SEGURANÇA DO PACIENTE EM UNIDADES BRASILEIRAS DE MÉDIO E ALTO RISCOS: REVISÃO SISTEMÁTICA DE LITERATURA
}

\section{PATIENT SAFETY IN BRAZILIAN MEDIUM AND HIGH RISK UNITS: SYSTEMATIC LITERATURE REVIEW}

\section{SEGURIDAD DEL PACIENTE EN UNIDADES BRASILEÑAS DE MEDIO Y ALTO RIESGO: REVISIÓN SISTEMÁTICA DE LA LITERATURA}

James Souza Santos ${ }^{1}$, Pamela Silverio De Lima ${ }^{2}$, Helen Cristiny Teodoro Couto ${ }^{3}$, Liliane de Lourdes Teixeira Silva ${ }^{4}$, Alba Otoni ${ }^{5}$.

\section{RESUMO}

Objetivo: avaliar a implantação de ferramentas, para a segurança (protocolo do programa nacional de segurança do paciente), na assistência ao paciente adulto e idoso internado em unidades brasileiras de médio e alto risco. Método: trata-se de uma revisão sistemática de literatura baseada na ferramenta Prisma (2015). As bases de dados PubMed, Scientific Eletronic Library On-line (SciELO) e biblioteca virtual de saúde (BVS) foram utilizadas para a busca dos artigos. Resultados: A análise conjunta dos estudos mostrou que as ferramentas, para a segurança, na assistência aos pacientes adultos e idosos internados, variaram entre as instituições brasileiras de médio e alto risco e são pontuais, não sendo identificada nenhuma unidade que adota todas as ferramentas de segurança do paciente. Conclusão: Os protocolos do programa nacional de segurança do paciente são ferramentas implantadas de forma pontual pelas unidades brasileiras de média e alta complexidade. Descritores: Segurança do Paciente; Assistência de Enfermagem; Instituições de Saúde.

\section{ABSTRACT}

Objective: To evaluate the implementation of safety tools (protocol of the National Patient Safety Program) in the care of adult and elderly patients admitted to Brazilian medium and high risk units. Method: This is a systematic literature review based on the Prisma tool (2015). The PubMed, Scientific Electronic Library Online (SciELO) and Virtual Health Library (VHL) databases were used to search the articles. Results: The joint analysis of the studies showed that the tools for safety in the care of hospitalized adult and elderly patients varied between medium and high-risk Brazilian institutions and are punctual; no unit that adopts all patient safety tools was identified. Conclusion: The protocols of the national patient safety program are tools implemented in a timely manner by Brazilian medium and high complexity units.

Keywords: Patient Safety; Nursing Care; Health Facilities.

\section{RESUMEN}

Objetivo: Evaluar la implementación de herramientas de seguridad (protocolo del Programa Nacional de Seguridad del Paciente) en la atención de pacientes adultos y ancianos ingresados en unidades brasileñas de riesgo medio y alto. Método: Esta es una revisión sistemática de la literatura basada en la herramienta Prisma (2015). Las bases de datos PubMed, Scientific Electronic Library Online (SciELO) y Virtual Health Library (VHL) fueron usadas para buscar los artículos. Resultados: El análisis conjunto de los estudios mostró que las herramientas para la seguridad en la atención de pacientes adultos y ancianos hospitalizados variaron entre las instituciones brasileñas de riesgo medio y alto y son puntuales, y no se identificó ninguna unidad que adopte todas las herramientas de seguridad del paciente. Conclusión: Los protocolos del programa nacional de seguridad del paciente son herramientas implementadas de manera oportuna por las unidades brasileñas de media y alta complejidad.

Palabras clave: Seguridad del Paciente; Cuidado de Enfermería; Instituciones de Salud.

${ }^{1}$ Graduando em Enfermagem pela Universidade Federal de São João del-Rei. ${ }^{2}$ Graduanda em Enfermagem pela Universidade Federal de São João del-Rei. ${ }^{3}$ Enfermeira. Doutora em Enfermagem pela Escola de Enfermagem da UFMG. Professora Adjunta na Universidade Federal de São João del-Rei. ${ }^{4}$ Enfermeira. Doutora em Ciências da Saúde Faculdade de Medicina UFMG. Professora Adjunta na Universidade Federal de São João del-Rei. ${ }^{5}$ Enfermeira. Doutora em Ciências da Saúde Faculdade de Medicina UFMG. Professora Adjunta na Universidade Federal de São João del-Rei.

Como citar este artigo:

Santos JS, Lima PS, Couto HCT, et al. Segurança do paciente em unidades brasileiras de médio e alto risco: revisão sistemática de literatura. Revista de Enfermagem do Centro-Oeste Mineiro. 2019;9:e3493. [Access ]; Available in: DOI: 


\section{INTRODUÇÃO}

A prestação de cuidados ao ser humano, inerente à enfermagem, traz inúmeros benefícios, mas também é passível de muitas ocorrências de erros. A complexidade dos novos tratamentos e as tecnologias do cuidar, além do aumento da carga de doenças da população, sem o devido planejamento e gerenciamento adequado do risco, podem contribuir para surgimento de danos importantes ao paciente que culminam com o aumento da permanência hospitalar, lesões, infecções e até mesmo a morte do enfermo ${ }^{(1)}$. Esses danos são consequências dos chamados Eventos Adversos (EA), que se caracterizam como incidentes que resultam em danos à saúde ${ }^{(1-3)}$.

A fim de prevenir e diminuir a frequência de EA e promover a segurança do paciente, no ano de 2013, foi instituído, no Brasil, o Programa Nacional de Segurança do Paciente (PNSP) com ações obrigatórias para todos os estabelecimentos de saúde e, dentre elas, a implantação de protocolos de segurança do paciente ${ }^{(2)}$. Esses protocolos são ferramentas para a implantação de medidas voltadas para a segurança do paciente e foram baseados em metas internacionais da Organização Mundial da Saúde (OMS). Foram elaborados pelo Ministério da Saúde (MS) seis protocolos: prática de higiene das mãos em estabelecimentos de saúde; cirurgia segura; segurança na prescrição/ uso e administração de medicamentos; identificação do paciente; prevenção de quedas; úlceras por pressão. Além dessas temáticas dos Protocolos, o PNSP abarca medidas à comunicação no ambiente dos estabelecimentos de Saúde e uso seguro de equipamentos e materiais ${ }^{(2,4)}$.

Tomando como referência os protocolos do MS e a atuação da equipe dos profissionais de enfermagem, entende-se que esses profissionais têm participação muito importante e direta, no alcance de uma assistência de qualidade, balizada em procedimentos seguros para o paciente. A posição ocupada pelo enfermeiro na assistência e, em consequência, as decisões de abordagem no cuidado, geram grande impacto na qualidade e excelência dos serviços prestados, podendo ser tomadas decisões assertivas ou não, que irão finalmente influenciar o risco de ocorrência de $E A^{(1-5)}$.

As instituições de saúde que não utilizam ferramentas, para a promoção da segurança, apresentam maiores riscos de ocorrência de EA e quanto maior o tempo de internação dos pacientes, maior será o risco de danos gerados pelos EA relacionados à assistência ${ }^{(6)}$.

Diante do exposto, confirma-se que a aplicação de ferramentas à promoção da segurança do paciente deve ser indissociável da prática clínica assistencial. No entanto a literatura, em especial, no Brasil, ainda é escassa sobre a real implementação e aplicação dessas ferramentas para a diminuição/prevenção dos EA nas instituições brasileiras. Assim, o objetivo deste estudo foi avaliar a implantação de ferramentas, para a segurança na assistência ao paciente adulto e idoso internado, em unidades brasileiras de médio e alto risco.

\section{MÉTODO}

Delineamento do estudo: trata-se de um estudo de revisão sistemática de literatura, baseada na ferramenta Prisma, que recomenda: formulação de um problema, revisão bibliográfica, seleção dos artigos, análise dos dados e apresentação da revisão ${ }^{(7)}$.

Critérios de elegibilidade: os critérios de elegibilidade foram elaborados, a partir da pergunta de pesquisa criada, considerando a estratégia "PICOS": "P"(pacients): pacientes adultos e idosos em internação hospitalar de ambos os sexos; "I" (intervention): prática de higiene das mãos em estabelecimentos de Saúde; cirurgia segura; segurança na prescrição/uso e administração de medicamentos; identificação do paciente; comunicação no ambiente dos estabelecimentos de Saúde; prevenção de quedas; úlceras por pressão; transferência de pacientes entre pontos de cuidado e uso seguro de equipamentos e materiais ${ }^{(2)}$. “ $\mathrm{C}$ " (control): pacientes que não foram atendidos com ações de segurança; "O" (outcomes): eventos adversos; "S" (study design), ensaios clínicos randomizados e observacionais (Transversal, Coorte e Casocontrole). Pergunta norteadora: "Quais ferramentas de segurança preconizadas pelo MS, na assistência aos pacientes internados, são efetivadas de fato nas instituições brasileiras de saúde?".

Assim, a busca incluiu artigos de ensaios clínicos randomizados e estudos observacionais realizados, no Brasil, até 14 de setembro de 2018. Não houve restrição de linguagem, nem de ano, para o alcance do maior número possível de estudos, sendo incluídos mesmo aqueles com mais de cinco anos. Os critérios de exclusão foram publicações, em formato de editorial, relatos de caso, opiniões de especialista e artigos 
de revisão e publicações que se refiram à segurança do paciente na atenção primária.

Fontes de Informação: a pesquisa sistemática foi realizada, utilizando as bases de dados PubMed, Scientific Eletronic Library On-line (SciELO) e Biblioteca Virtual de Saúde (BVS). Busca: a estratégia de pesquisa foi realizada, utilizando os descritores cadastrados no $\mathrm{MeSH}$ e DeCs, e o operador booleano "AND" foi utilizado, para fazer a associação dos termos, em todas as bases de dados: (1) "Patient safety"(MeSH) AND "nursing"; (2) "segurança do paciente" AND "Assistência de enfermagem".

Extração de dados: todos os artigos foram avaliados por dois revisores (J.S.S e P.S.D.L). Em um primeiro momento, foram removidos os artigos duplicados. A seguir, na fase de avaliação dos artigos, realizou-se a leitura de títulos e resumos. Foram excluídos os artigos que não estavam de acordo com os critérios de elegibilidade e aqueles selecionados foram submetidos à segunda fase de análise, a qual consistiu na leitura do texto completo e dado um novo parecer por cada revisor para inclusão ou exclusão no artigo atual. Aqueles que tiveram discordância, quanto à inclusão ou exclusão, foram novamente lidos e mantiveram-se ou foram excluídos, conforme o consenso da dupla revisora (FIGURA 1).

Figura 1 - Seleção dos artigos por estratégia de busca nas bases de dados. Divinópolis (MG), Brasil, 2019.

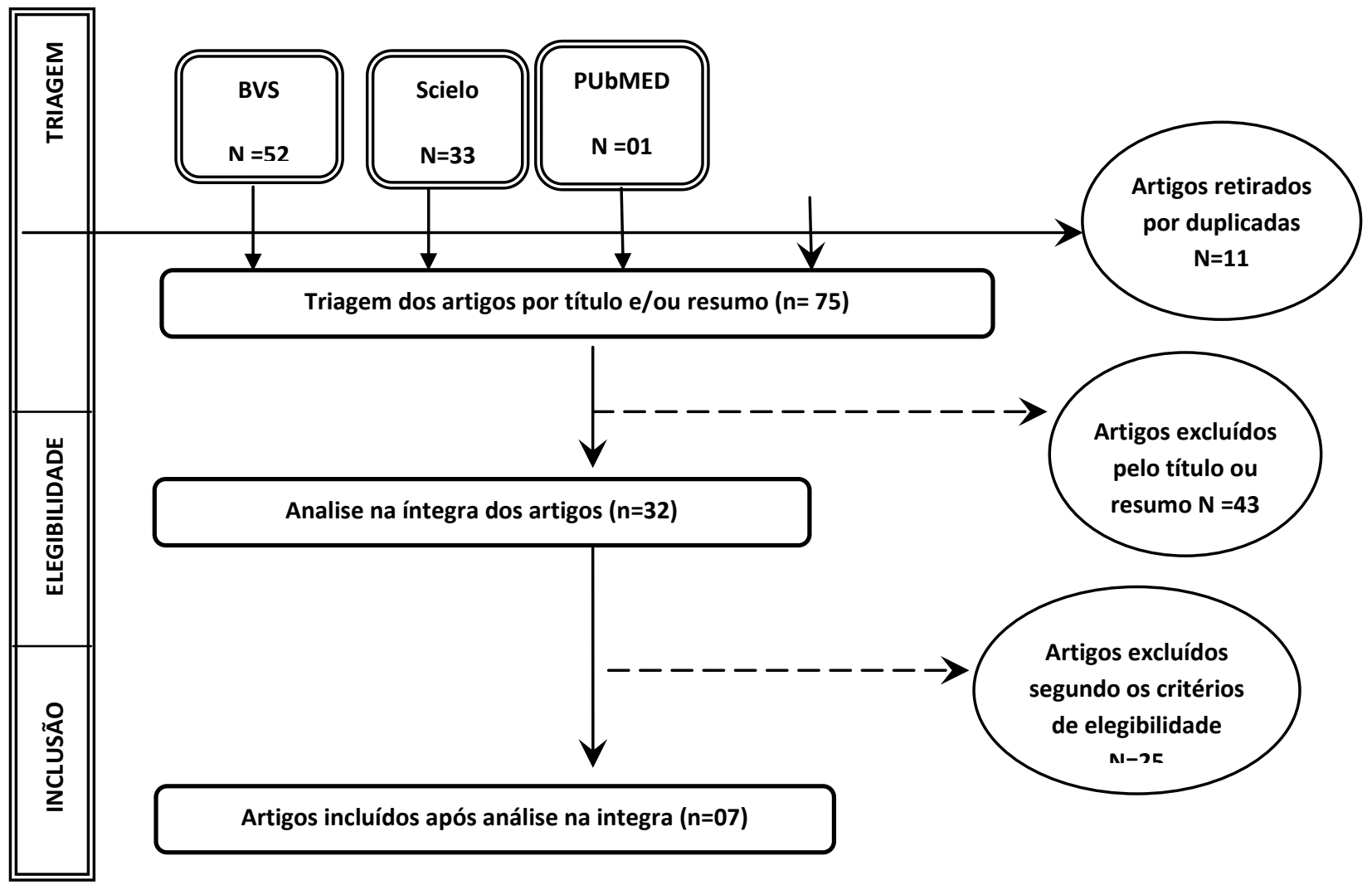

Fonte: Dados da pesquisa, 2018.

As seguintes variáveis foram extraídas dos artigos selecionados para análise: (1) autor, ano de publicação, país de publicação; (2) participantes e tamanho da amostra; (3) Critérios para definir ações de segurança do paciente; (4) principais resultados (TABELA 1).

\section{RESULTADOS E DISCUSSÃO}

No total, 86 artigos foram selecionados, na busca, em todas as bases de dados descritas.
Após a remoção dos duplicados, restou um total de 75 artigos que foram avaliados pelos títulos e resumos. Por meio dos critérios de elegibilidade propostos, sete artigos foram selecionados e lidos na íntegra. Entre esses, um artigo era de pesquisa-ação, um artigo de coorte, uma pesquisa exploratória e quatro estudos descritivos (sendo um de abordagem descritivoanalítico-qualitativa, um corte transversal, um descritivo retrospectivo e um descritivo 
Tabela 1 - Estudos sobre ferramentas, para a segurança, na assistência ao paciente adulto e idoso, em instituições brasileiras de médio e alto risco (" $\mathrm{N}$ " de Profissionais de saúde=67; " $\mathrm{N}$ " de docentes e discentes universitários=162; " $\mathrm{N}$ " de pacientes= 2.256; “ $\mathrm{N}$ " total da revisão $=2485$ pessoas)

\begin{tabular}{|c|c|c|c|}
\hline & Autor/Ano/País & $\begin{array}{c}\text { Participantes/Tamanho } \\
\text { da amostra }\end{array}$ & $\begin{array}{c}\text { Critérios para definir ações de } \\
\text { segurança do paciente. }\end{array}$ \\
\hline 1 & $\begin{array}{l}\text { Paranaguá, et al., } \\
2014\end{array}$ & Pacientes $=735$ & $\begin{array}{l}\text { Instrumento estruturado } \\
\text { contendo assertivas sobre a } \\
\text { temática pesquisada }\end{array}$ \\
\hline 2 & $\begin{array}{l}\text { Paixão, et al., } \\
\text { 2018, Brasil }\end{array}$ & Pacientes $=377$ & $\begin{array}{l}\text { Instrumento de verificação de } \\
\text { ações básicas de segurança } \\
\text { validado por especialistas } \\
\text { brasileiros }\end{array}$ \\
\hline 3 & $\begin{array}{c}\text { Belela-Anacleto, } \\
\text { et al., 2013, } \\
\text { Brasil }\end{array}$ & $\begin{array}{c}\text { Docentes }=53 \\
\text { Discentes }=109\end{array}$ & $\begin{array}{l}\text { Instrumento estruturado } \\
\text { contendo assertivas sobre a } \\
\text { temática pesquisada }\end{array}$ \\
\hline 4 & $\begin{array}{l}\text { Oliveira, et al., } \\
\text { 2017, Brasil }\end{array}$ & Pacientes $=96$ & $\begin{array}{l}\text { Aplicação da escala "Fall Risk } \\
\text { Score" para a identificação do } \\
\text { alto risco para queda }\end{array}$ \\
\hline
\end{tabular}

5 Sakai, et al., 2016, Brasil

Pacientes $=1408$

6

Oliveira, et al, 2014, Brasil

Enfermeiros $=37$
A coleta foi realizada, por meio de auditoria operacional, para a avaliação de 15 indicadores de qualidade assistencial, dentre os quais está o indicador "Risco de Queda"

Roteiro de entrevista semiestruturada composta de questões norteadoras sobre conceitos, critérios de avaliação da estrutura física, humana e organizacional necessária à promoção da segurança do paciente, além dos riscos relacionados à assistência de enfermagem.
Principais resultados

Entre os incidentes relacionados aos cuidados, destacaram-se os referentes à medicação e à omissão de cuidados.

Evidenciou-se a ausência de identificação sistemática dos pacientes, avaliação e sinalização do risco de queda e desenvolvimento de lesões por pressão

As atividades de estágio ocorrem em locais, nos quais não há adequada infraestrutura, para práticas preconizadas de $\mathrm{HM}^{*}$, fato que contribui à ocorrência de falhas, no processo de cuidar e compromete a segurança do paciente

O estado psicológico, o uso de órtese e o déficit cognitivo foram identificados como fatores de risco para queda em idosos hospitalizados

O escore de alto risco de queda foi mais prevalente nas unidades médico-cirúrgicas. As medidas de prevenção estavam adequadas em $91,0 \%$. Conhecer os riscos de queda e os indicadores de qualidades, associados com a sensibilização e capacitação dos profissionais, são medidas imprescindíveis na prevenção de incidentes e eventos adversos.

Os participantes identificaram riscos físicos/químicos, clínicos, assistenciais e institucionais, além de barreiras e oportunidades que implicam a (in) segurança do paciente. Por outro lado, referiram-se a práticas embasadas, em metas internacionais, divulgadas pela Organização Mundial de Saúde.

Os profissionais não perceberam mudanças na comunicação interpessoal com o uso do checklist, porém indicaram que o uso proporcionou mais segurança ao procedimento. Apontaram, ainda, necessidade de mudanças, no checklist, para garantir cirurgias seguras 
Dos sete estudos selecionados, a população envolvida foi de 67 profissionais de saúde; 53 docentes e 109 discentes universitários e, também, de 2.256 pacientes submetidos aos cuidados em unidades de saúde. Incluíram-se participantes de ambos os sexos, adultos e idosos totalizando uma população total de 2485 .

Resgatando o primeiro artigo selecionado, em estudo transversal desenvolvido por Paranaguá et al. ${ }^{(8)}$, o objetivo foi estimar a prevalência e fatores associados à ocorrência de incidentes relacionados à medicação, registros nos prontuários dos pacientes internados na clínica cirúrgica de um hospital de ensino. Neste estudo, identificou-se que a maior ocorrência de falha, na aplicação do protocolo de segurança na prescrição, uso e administração de medicamentos, estava relacionada à omissão da dose e ausência de checagem de medicamentos. Além disso, evidenciou-se ainda óbito como a consequência mais grave ocorrida.

Paixão et al. ${ }^{(9)}$, em 2018, ao realizarem um importante estudo exploratório com 377 participantes, investigaram o cumprimento dos protocolos nacionais de segurança do paciente em Unidade de Pronto Atendimento (UPA) no Paraná. Nos resultados, as categorias: a) identificação dos pacientes; b) avaliação de pacientes suscetíveis à queda e desenvolvimento de lesão por pressão e c) a identificação de soluções administradas foram as que mais apresentaram fragilidades, quanto à adesão das ações básicas de segurança do paciente. Ressaltase, também, que indivíduos que pertenciam aos grupos geriátricos e pediátricos manifestaram maior risco e probabilidade para a ocorrência de eventos adversos (EA).

Belela-Anacleto et al. ${ }^{(10)}$ desenvolveram estudo com o objetivo de identificar a perspectiva de docentes e universitários da área da saúde sobre aspectos relacionados à higienização das mãos e infecções relacionadas à assistência à saúde, no cotidiano de sua prática. Esse estudo avaliou uma população de 109 universitários e 53 docentes de uma universidade pública de São Paulo, Brasil. A partir dos seus resultados, puderam identificar que a melhor forma de prevenção das infecções, relacionadas à assistência à saúde (IRAS), foi a aplicação do protocolo de segurança higienização das mãos (HM).

Mesmo assim, segundo os autores, na opinião dos docentes e universitários da área da saúde, embora esteja clara a importância da higienização das mãos, como medida de segurança, as atividades de estágio ainda ocorrem em locais, nos quais não há adequada infraestrutura, para as práticas preconizadas de $\mathrm{HM}$, fato que contribui, para a ocorrência de falhas, no processo de cuidar e compromete a segurança do paciente. Oliveira et al. ${ }^{(11)}$ e Sakai et al. ${ }^{(12)}$ investigaram o protocolo de segurança "risco de queda e medidas de prevenção". Ambos os estudos encontraram que indivíduos idosos estão mais vulneráveis ao risco de queda. Oliveira et al. ${ }^{(11)}$ destacam que, dentre os idosos que apresentaram queda, a maioria era do sexo feminino, com idade média de 74,81 anos. 0 estado psicológico, o uso de órtese e o déficit cognitivo foram identificados como fatores de risco para queda em idosos hospitalizados. Sakai et al. ${ }^{(12)}$ identificaram que pacientes com idade superior a 60 anos apresentaram maior risco de queda $(33,9 \%)$. Os indivíduos classificados com alto risco foram os que estavam conectados a dispositivos venosos $(90,1 \%)$, os que apresentaram histórico de queda anterior $(59,9 \%)$ e com estado mental desorientado (34,0\%). As medidas de prevenção estavam adequadas em $91,0 \%$.

Outro estudo de Oliveira et al. ${ }^{(13)}$, cujo objetivo foi identificar e analisar estratégias, para promover a segurança do paciente, na perspectiva de enfermeiros, identificou que riscos físico-químicos, clínicos, assistenciais e institucionais foram os fatores que implicaram a (in) segurança do paciente $\mathrm{e}$, portanto precisariam ser trabalhados a fim de tornar a assistência segura. Ademais, revelaram que havia uma necessidade de atenção dos gestores ao incentivo e capacitação dos profissionais de saúde, para a prevenção, notificação e manejo efetivo dos riscos de EA durante a realização e a avaliação da assistência prestada.

Pancieri et al. ${ }^{(14)}$, em seu estudo de campo, realizado em um hospital-escola, aplicaram um "checklist de cirurgia segura" com o objetivo de analisar a sua contribuição para a segurança do processo cirúrgico. Os resultados identificaram o instrumento como viável para garantir cirurgias seguras e implementar processos comunicativoefetivos nesses ambientes. Deixaram claro, também, que a aplicação do "checklist de cirurgia segura", em Centro Cirúrgico (CC), mostrou uma melhor eficácia, proporcionando maior segurança, durante os procedimentos, além de favorecer a interação e a comunicação entre a equipe. Uma das dificuldades encontradas à 
implantação do checklist foi a aceitação do método por todos os profissionais.

Nos estudos elencados para esta revisão, ficou evidenciado que as ferramentas, para a segurança dos pacientes, variam entre as instituições brasileiras de médio e alto risco, não havendo um perfil-padrão de sua implementação. Os dados disponíveis na literatura foram limitados, no sentido de permitir a confirmação de implementação de "todas" as ferramentas de segurança à assistência, nas unidades de médio e alto risco, conforme recomenda a RDC $2013^{(3)}$. E, embora esse seja um tema discutido, há algumas décadas, somente foi regulamentado pelos órgãos responsáveis, há seis anos, com definição mais precisa de ações que irão garantir uma assistência segura. Após esses seis anos de regulamentação de medidas específicas, para uma assistência segura, acredita-se que haja uma vivência maior com a implementação de ferramentas de segurança nas instituições de médio e alto risco e, por isso, é provável que aumente o número de estudos que possam evidenciar, de forma mais padronizada, como se encontram as aplicações práticas de assistência segura em instituições brasileiras de médio e alto risco.

No estudo desenvolvido por Paranaguá et al. ${ }^{(8)}$, a maior falha de segurança na assistência ficou centralizada, em uma atividade de rotina da equipe de enfermagem, a administração de medicamentos. Nesse estudo, além de identificarem erros de doses dos medicamentos, a falta de checagem, após sua administração, comprometeu significativamente a segurança do paciente. $E$, muito importante, também, foi a identificação, nesse estudo, de óbitos como consequências a uma prática não segura de assistência. Resultados que demonstram que esse tipo de comprometimento do estado de saúde dos pacientes, em razão da prática não segura de assistência, são muito impactantes e precisam ser repensados na prática clínica da assistência de enfermagem. Para além da identificação das metas de segurança não aplicadas, Paranaguá et $\mathrm{al}^{(8)}$ discutiram, ainda, a falta de notificação de EA como fator dificultador, na tomada de decisão, para medidas corretivas e orientadoras a condutas futuras. Como alternativa, a fim de minimizar a situação encontrada, sugeriram a criação de instrumentos-padrões que facilitassem essas notificações, além da aplicação de medidas não punitivas a fim de oferecer segurança ao profissional, ao relatar o EA com riqueza de detalhes que possibilitasse a correção das falhas na assistência. O contexto de segurança do paciente, neste estudo, foi comprometido, basicamente, em função das condutas adotadas pela equipe de enfermagem, que aparentemente não se sentiu segura ao relatar a ocorrência do $E A$. Ao se identificar um EA, que põe em risco a vida do paciente, é preciso muita maturidade profissional e conduta ética, para expor a situação e cooperar com nas ações sequenciais, em busca do aprendizado, para que aquele evento não mais ocorra. Entende-se, porém que, mesmo diante das possíveis consequências administrativas e profissionais, a notificação do EA deve ser, sim, a conduta-padrão da equipe de enfermagem, não devendo esses eventos serem vistos como "naturais" do exercício profissional.

Ainda, em se tratando de administração de medicamentos, embora Paixão et al. ${ }^{(9)}$ não tenham classificado quais as metas seriam avaliadas, em seu estudo na UPA, ao descreverem entre as categorias que a falta de identificação, em soluções administradas, foi um indicador da falta de segurança, sugeriram a falha no cumprimento do protocolo "segurança na prescrição, uso e administração de medicamentos" como fragilidade na aplicação de medidas de segurança aos pacientes assistidos na UPA. Esses tipos de instituições de saúde atendem pacientes considerados mais graves, agudos e, em situação de risco de vida e, por isso, a assistência, sem o uso das devidas ferramentas de segurança, podem trazer, na maioria das vezes, consequências muito graves ao paciente. A base do planejamento assistencial deve ser a segurança do paciente, não podendo, de forma alguma, ser vista como uma ação complementar à assistência.

Uma medida de segurança, teoricamente mais simples, porém, com potencialidade para impactar de forma a comprometer a integridade física do paciente, é a higienização das mãos. Belela-Anacleto et al. ${ }^{(10)}$ avaliaram a perspectiva de docentes e universitários da área da saúde, nos campos de estágio sobre aspectos de higienização das mãos e infeç̧ões relacionadas à assistência à saúde, no cotidiano da prática assistencial. De forma muito clara, os resultados desse estudo mostraram que os docentes e discentes voltaram a confirmar o que a precursora da enfermagem moderna, Florence Nightingale, já falava desde o século passado: a higienização das mãos pode evitar a instalação de quadros infecciosos e a não efetivação desta ação 
de segurança compromete de forma importante a integridade física do paciente assistido. Essa constatação parece quase impossível ou mesmo fora da realidade, quando se identifica a assistência ao paciente sem a HM. Porém, sem justificar, mas explicando, os autores identificaram que, em muitas situações, a falta de HM pode ocorrer, não necessariamente pela falta de compromisso dos profissionais, mas pela falta de infraestrutura mínima, nos locais, onde se realizam os estágios (disponibilização de pias, por exemplo). Acredita-se que, talvez, essa falta de infraestrutura mínima seja consequência de uma cultura nacional por parte dos nossos gestores públicos de que a saúde não é um bem primordial a ser preservado e, portanto não é vista como prioridade de investimento do dinheiro público. Esse cenário de "crise na saúde" a que o Brasil vem sendo exposto, historicamente, parece fomentar condutas desfavoráveis à assistência que variam desde questões administrativas até a assistência direta propriamente dita.

Dois outros autores estudados, nesta revisão, Oliveira et al. ${ }^{(11)}$ e Sakai et al. ${ }^{(12)}$ investigaram 0 protocolo de segurança "Prevenção de quedas" e medidas de prevenção. Para Oliveira et al. ${ }^{(11)}$, estão mais predisponentes ao desenvolvimento do evento aqueles indivíduos conectados a dispositivos venosos, os que já apresentaram um histórico de queda anterior e aqueles que apresentam alterações neurológicas. Sankai et al. ${ }^{(12)}$ salientaram que indivíduos, que apresentam um déficit cognitivo, os diagnosticados com depressão ou os portadores de órteses estão mais suscetíveis. Neste sentido, pode-se afirmar que esses autores identificaram um padrão de pacientes mais susceptíveis à queda, que é outro indicador de segurança do paciente. E reconhecer os riscos de queda é a melhor forma de a equipe multidisciplinar estabelecer estratégias, para a manutenção da saúde desses indivíduos, diminuindo os EA. Por isso, é tão importante a aproximação e interação entre todos os profissionais da saúde que promovem a assistência, no sentido de reconhecer a realidade, incluindo os riscos a que o paciente está exposto, para que se possa trabalhar conjuntamente medidas de prevenção adequadas ao perfil de cada paciente.

Oliveira et al. ${ }^{(13)}$ corroboram os autores citados e destacam que a melhor forma de prestar uma assistência segura e livre de danos é, por meio do levantamento dos riscos aos quais os indivíduos estão expostos, durante a assistência de enfermagem, sendo assim, trabalhar estratégias que colaborem para extinguir o risco dos EA. Salienta-se, no entanto que todas essas ações de segurança dependem também das condições de trabalho do profissional da saúde. É necessário garantir condições adequadas ao desenvolvimento eficiente das atividades de rotina, preservando a dignidade profissional, favorecendo, em consequência, uma assistência de excelência.

O protocolo "cirurgia segura" foi identificado, no estudo de Pancieri et al. ${ }^{(14)}$, em que os pesquisadores mostraram que $50 \%$ das causas de EA, em procedimentos cirúrgicos, poderiam ser evitados, se fossem trabalhadas adequadamente medidas de segurança. Foi demonstrado que a aplicação do checklist de cirurgia segura, em centros cirúrgicos, favoreceu - sucesso em procedimentos cirúrgicos realizados, além de ter facilitado a interação e comunicação entre a equipe. Interessantemente destacaram que uma das dificuldades encontradas, na implantação do checklist, foi a aceitação do método pelos profissionais envolvidos. Novamente parece paradoxal que pessoas envolvidas na assistência tenham resistência a implementar medidas que irão garantir o bem-estar e integridade física do paciente. Entende-se que o enfermeiro, neste momento, tem papel fundamental como gestor, na sensibilização da equipe e conscientização de que trabalhar com medidas de segurança não é uma escolha do profissional de saúde assistencial, mas um dever inerente a toda e qualquer prática de assistência.

Por fim, embora esta revisão tenha demonstrado a realidade das medidas de segurança, nas unidades hospitalares, contribuindo para um desvelamento atual dessa realidade, apresenta limitações inerentes ao desenvolvimento de algumas revisões: não foi possível padronizar o delineamento de estudo a ser incluído, exatamente, pela escassez de publicações científicas nesta área.

Além disso, não foi possível avaliar a qualidade metodológica dos estudos, por não haver instrumento validado, que permitisse a avaliação de todos os tipos de delineamentos que foram incluídos nesta revisão. De qualquer forma, acredita-se que este estudo trouxe importantes resultados que poderão ser utilizados para aprimorar a assistência em enfermagem a pacientes hospitalizados. 


\section{CONSIDERAÇÕES FINAIS}

Os protocolos do programa nacional de segurança do paciente são ferramentas implantadas de forma pontual pelas instituições brasileiras de médio e alto risco, não sendo identificada nenhuma instituição que tenha registrado a implantação de todas as ferramentas de segurança na assistência ao paciente. Vale ponderar que a escassez de artigos sobre segurança do paciente específicos, em unidades brasileiras de médio e alto risco, ainda, é bastante proeminente e, por isso, foram inseridos nesta revisão artigos com mais de cinco anos.

De qualquer maneira, ficou claro que a melhor forma de prestar uma assistência livre de EA é por meio do gerenciamento dos riscos aos quais está exposto o indivíduo em estado de adoecimento. Ressalta-se que os protocolos são ferramentas direcionadoras que têm o objetivo de auxiliar na mitigação e prevenção da ocorrência de EA. Recomenda-se também que as instituições de saúde se sensibilizem e compreendam a importância da notificação correta dos casos ocorridos, nas unidades de atendimento e, por meio dessas notificações, realizem ações educativas e não punitivas, ressaltando o quanto é significativo tanto para o paciente quanto para o profissional uma assistência livre de danos.

\section{REFERÊNCIAS}

1- Siman AG, Cunha SGS, Brito MJM. The practice of reporting adverse events in a teaching hospital. Rev Esc Enferm USP 2018;51:1-8. DOI: $\underline{10.1590 / s 1980-220 \times 2016045503243}$

2- Brasil, Ministério da Saúde. Portaria no 529, de 1 de abril de 2013. Institui o Programa Nacional de Segurança do Paciente. Diário Oficial da União 2013a.

3- Brasil, Ministério da Saúde. Resolução da Diretoria Colegiada - RDC no 36, de 25 de julho de 2013. Institui ações para a segurança do paciente em serviços de saúde e dá outras providências. Diário Oficial da União 2013b.

4- Brasil, Ministério da Saúde. Documento de referência para o Programa Nacional de Segurança do Paciente. Brasília: Ministério da Saúde; 2014.

5- Chaves LDP, Mininel VA, Silva JAM, Alves LR, Silva MF, Camelo SHH. Nursing supervision for care comprehensiveness. Rev Bras Enferm. 2017;70(5):1106-11. DOI: 10.1590/0034-7167$\underline{2016-0491}$
6- Roque KE, Tonini T, Melo EC. Adverse events in the intensive care unit: Impact on mortality and length of stay in a prospective study. Cad Saúde Pública 2016;32(10):1-15. DOI: 10.1590/0102-311X00081815

7- Liberati A, Altman DG, Tetzlaff J, Mulrow C, Gøtzsche PC, loannidis JPA, et al. The PRISMA statement for reporting systematic reviews and meta-analyses of studies that evaluate health care interventions: Explanation and elaboration. PLoS Med. 2009;6(7): e1000100. DOI: 10.1371/journal.pmed.1000100

8- Paranaguá TTB, Bezerra ALQ, Santos ALM, Silva AEBC. Prevalence and factors associated with incidents related to medication in surgical patients. Rev Esc Enferm USP 2014;48(1):41-7. DOI: 10.1590/S0080-623420140000100005

9- Paixão DPSS, Batista J, Maziero ECS, Alpendre FT, Amaya MR, Cruz EDA. Adhesion to patient safety protocols in emergency care units. Rev Bras Enferm. 2018;71(Suppl 1):577-84. DOI: 10.1590/0034-7167-2017-0504

10- Belela-Anacleto ASC, Sousa BEC, Yoshikawa JM, Avelar AFM, Pedreira MLG. Hand hygiene and patient safety: Perspectives of professors and undergraduate students. Texto Contexto-Enferm. 2013;22(4):901-8. DOI: 10.1590/S0104$\underline{07072013000400005}$

11- Oliveira DU, Ercole FF, Samara ML, Matos SSD, Campos CC, Fonseca EM. Evaluation of falls in hospitalized elderly. Rev Enferm UFPE 2017;11(11):4589-97. DOI: 10.5205/1981-8963v11i11a231198p4589-4597-2017

12- Sakai AM, Rossaneis MA, Haddad MDCFL, Vituri DW. Risk of bed falls in adult patients and prevention measures. Rev Enferm UFPE 2016;10(6):4720-26. DOI: 10.5205/1981-8963v10i6a11249p4720-4726-2016

13- Oliveira RM, Leitão IM, Silva LM, Figueiredo SV, Sampaio RL, Gondim MM. Strategies for promoting patient safety: From the identification of the risks to the evidence-based practices. Esc Anna Nery 2014;18(1):122-9. DOI: 10.5935/1414$\underline{8145.20140018}$

14- Pancieri AP, Santos BP, Avila MAG, Braga EM. Checklist de cirurgia segura: Análise da segurança e comunicação das equipes de um hospital escola. Rev Gaúcha Enferm. 2013;34(1):71-8. DOI: $\underline{10.1590 / \text { S1983-14472013000100009 }}$

Nota: Nota: Trata-se do trabalho de conclusão do curso de enfermagem da Universidade Federal de São João del- Rei dos alunos James Souza Santos e Pâmela Silvério de Lima do curso. 
Recebido em: 24/06/2019

Aprovado em: 14/11/2019

Endereço de correspondência:

Alba Otoni

Universidade Federal de São João del-Rei. Rua: Sebastião

Gonçalves Coelho, 400 - Chanadour

CEP: 35501-296 - Divinópolis/MG - Brasil

E-mail: albaotoni@ufsj.edu.br 